\title{
A Study on Automatic Methods Based on Mathematical Morphology for Martian Dust Devil Tracks Detection
}

\author{
Thiago Statella ${ }^{1}$, Pedro Pina ${ }^{2}$, and Erivaldo Antônio da Silva ${ }^{3}$ \\ ${ }^{1}$ Instituto Federal de Educação, Ciência e Tecnologia de Mato Grosso - IFMT \\ 95 Zulmira Canavarro 780025-200, Cuiabá, Brazil \\ thiago.statella@cba.ifmt.edu.br \\ ${ }^{2}$ Centro de Recursos Naturais e Ambiente, Instituto Superior Técnico - IST \\ Av. Rovisco Pais 1049-001, Lisboa, Portugal \\ ppina@ist.utl.pt \\ ${ }^{3}$ Universidade Estadual Paulista, Faculdade de Ciências e Tecnologia - FCT \\ 305 Roberto Simonsen 19060-900, Presidente Prudente, Brazil \\ erivaldo@fct.unesp.br
}

\begin{abstract}
This paper presents three methods for automatic detection of dust devils tracks in images of Mars. The methods are mainly based on Mathematical Morphology and results of their performance are analyzed and compared. A dataset of 21 images from the surface of Mars representative of the diversity of those track features were considered for developing, testing and evaluating our methods, confronting their outputs with ground truth images made manually. Methods 1 and 3 , based on closing top-hat and path closing top-hat, respectively, showed similar mean accuracies around $90 \%$ but the time of processing was much greater for method 1 than for method 3. Method 2, based on radial closing, was the fastest but showed worse mean accuracy. Thus, this was the tiebreak factor.
\end{abstract}

Keywords: Mars, Dust Devils Tracks, Mathematical Morphology, Feature Detection.

\section{Introduction}

Dust devils are vortexes caused by unstable wind convection processes near the planetary surfaces, due to solar heat. They have been studied on Earth for more than a century and were first observed on Mars in orbital images taken by the Viking program in the 1970s. These phenomena can achieve miles in width and height, and knowledge about their activity contributes to the understanding of Martian climate, geology and surface modification which is essential to plan future manned missions $[1,2]$. According to [3], air circulation is one of the currently active processes which model the surface of Mars and some researches show that these vortexes are responsible for most of the linear and curvilinear surface features of the planet. Moreover, the inference of the wind direction based on dust devils tracks detection is one of the few techniques for verifying circulation models of the atmosphere. This fact suggests that more research on aeolian processes is needed. The direction of dust devils tracks can be used to get information on wind circulation, and it can be done by image analysis [3]. 
Many researchers have being studying dust devils in an attempt to better understand the phenomena. Generally, the research fields comprise mechanic and numerical simulation of dust devils in laboratories [4-6], methodologies for direct recognition of dust devils plumes from rovers on Mars surface [7-9], detection of plumes [10-12] and tracks [1, 3, 13, 14] from orbital images. Despite the number of papers regarding the subject, none of them addresses the automatic detection of dust devils tracks. All those works regarding identification, counting and analysis of dust devils tracks use a manual method and nothing is said about the tracks counting and marking process. As the number of well succeed missions launched to study Mars rises, so does the number of orbital images and their resolutions. There are hundreds of high resolution images depicting Martian surface, providing important data for, among others, researches in Geology, Cartography and Aeolian processes monitoring, at a level of detail never achieved before [15]. The amount of images taken (and therefore the amount of information on them) grew at a rate greater than the human capability to analyze and extract relevant information from these products to characterize the planet under study [16]. As examples of the difficulty in analyzing manually so many images, $[1,3,13,14,17]$ had to search for tracks in $(1,700)$, $(3,000),(6,002),(167,254)$ and $(1,238)$ MOC images, respectively; [10] searched in (23) HRSC images and [18] did that in $(3,079)$ THEMIS images.

Regarding the amount of images to be analyzed and the importance of detecting dust devils tracks, this paper presents, analyses and compares three automatic methods for detecting dust devils tracks in Mars Global Surveyor (MGS) Mars Orbiter Camera (MOC) and Mars Reconnaissance Orbiter (MRO) High Resolution Imaging Science Experiment (HiRISE) images. All three methods are mainly based on Mathematical Morphology.

\section{Image Datasets}

Based on some evidences [1, 3, 14] that dust devils are more likely to occur in the southern hemisphere and on the fact that they form during spring and summer, a search for MOC narrow angle (http://www.msss.com/msss_images/) and HiRISE (http://www.hirise.lpl.arizona.edu/) images with solar longitudes ranging between $180^{\circ}$ and $360^{\circ}$ containing tracks of dust devils was performed in the regions Aeolis, Noachis and Argyre.

A total of 16 images from those regions (12 MOC narrow angle and 4 HiRISE) showing dark dust devils tracks were considered. The albedo of the tracks varies significantly from scene to scene as does the morphology and landform. Based on that, the choice for these 16 images was driven by the attempt to represent as much as possible the high albedo variability of the tracks. In order to decrease time of processing and discard irrelevant information (like areas with no tracks) some of the images were cut, making a set of 21 images. Fig. 1 shows these images and Table 1 summarizes their characteristics. The MOC images are panchromatic and can have up to $1.4 \mathrm{~m} /$ pixel of spatial resolution and the HiRISE images were taken in the red band with a spatial resolution as good as $0.25 \mathrm{~m} /$ pixel. 


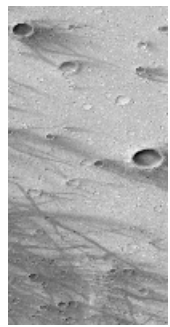

(a)

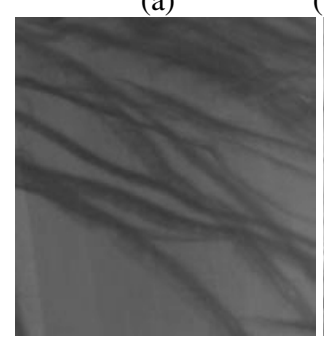

(f)

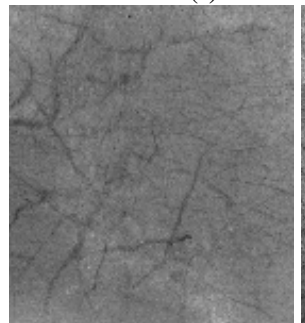

(k)

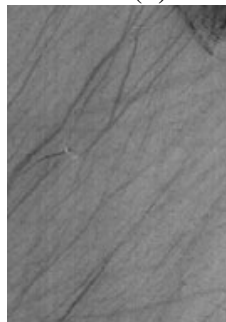

(p) (b)

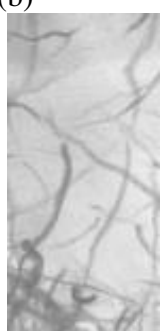

(g)

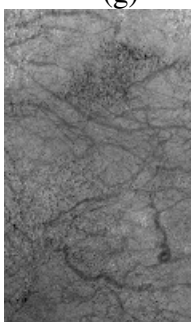

(1)

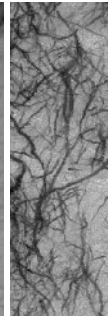

(q)

(r)

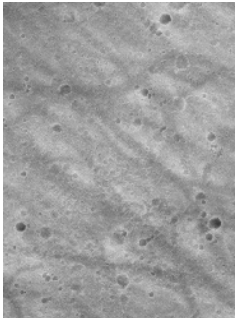

(c)

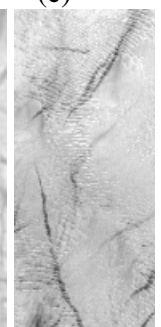

(h)

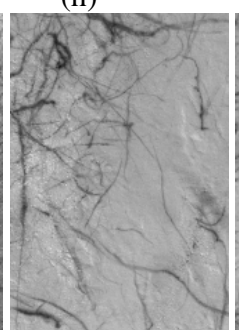

(m)

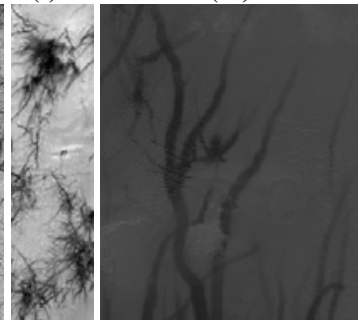

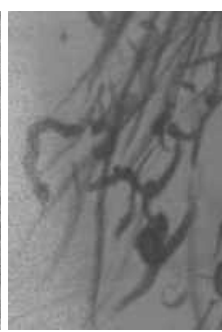

(d)

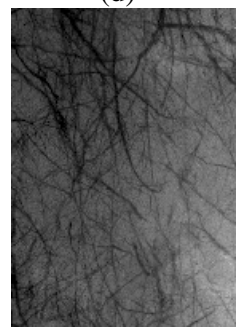

(i)

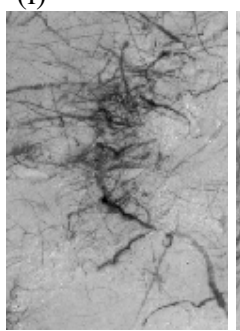

(n)

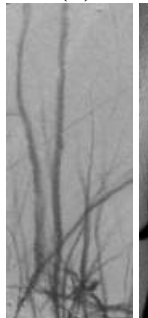

(t)

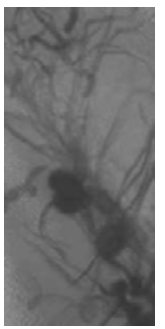

(e)

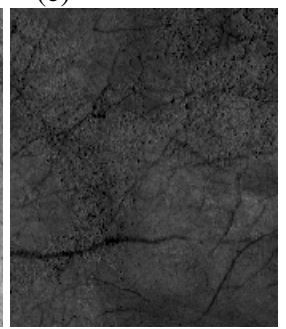

(j)

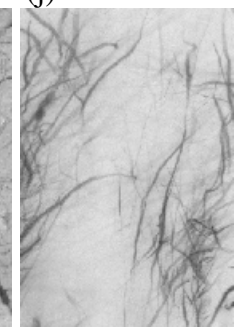

(o)

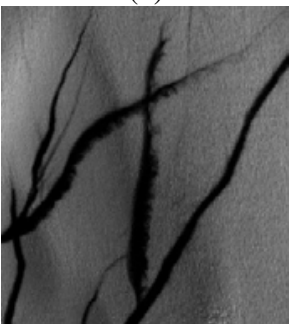

(u)

Fig. 1. Diversity of Martian dust devils tracks on MOC and HiRISE images. Table 1 shows information about each of them. 
Table 1. Summarized information about the images shown in Fig. 1. In the Table, "Res." stands for "Spatial Resolution" and letters (a) through (u) in the field "Name" correspond to the images shown in Fig. 1.

\begin{tabular}{cccccc}
\hline Name & Sensor & Date & Res. (m) & Size (pixels) & Region \\
\hline R02-00357(a) & MOC & $02 / 07 / 2003$ & 2.98 & $1,024 \times 2,052$ & Aeolis \\
R08-02402(b) & MOC & $08 / 27 / 2003$ & 1.50 & $1,856 \times 3,017$ & Aeolis \\
R13-01467(c) & MOC & $01 / 10 / 2004$ & 1.43 & $2,077 \times 2,855$ & Aeolis \\
PSP_002548_1255A(d) & HiRISE & $02 / 10 / 2007$ & 0.25 & $4,069 \times 2,779$ & Noachis \\
PSP_002548_1255B(e) & HiRISE & $02 / 10 / 2007$ & 0.25 & $5,582 \times 2,621$ & Noachis \\
PSP_002548_1255C(f) & HiRISE & $02 / 10 / 2007$ & 0.25 & $2,840 \times 3,011$ & Noachis \\
PSP_005528_1255(g) & HiRISE & $10 / 01 / 2007$ & 0.25 & $3,000 \times 1,432$ & Noachis \\
M10-01206(h) & MOC & $12 / 11 / 1999$ & 5.55 & $363 \times 829$ & Argyre \\
S08-02952(i) & MOC & $07 / 27 / 2005$ & 5.95 & $443 \times 529$ & Argyre \\
R13-02691(j) & MOC & $01 / 17 / 2004$ & 5.81 & $414 \times 590$ & Argyre \\
R08-02621A(k) & MOC & $08 / 30 / 2003$ & 4.40 & $564 \times 632$ & Argyre \\
R08-02621B(l) & MOC & $08 / 30 / 2003$ & 4.40 & $500 \times 856$ & Argyre \\
E13-00271A(m) & MOC & $02 / 03 / 2002$ & 5.83 & $462 \times 316$ & Argyre \\
E13-00271B(n) & MOC & $02 / 03 / 2002$ & 5.83 & $420 \times 606$ & Argyre \\
S10-01598(o) & MOC & $09 / 29 / 2005$ & 2.92 & $565 \times 841$ & Argyre \\
S08-03151(p) & MOC & $07 / 30 / 2005$ & 4.46 & $233 \times 339$ & Argyre \\
M14-00175A(q) & MOC & $04 / 03 / 2000$ & 5.53 & $349 \times 1,144$ & Argyre \\
M14-00175B(r) & MOC & $04 / 03 / 2000$ & 5.53 & $454 \times 1,795$ & Argyre \\
M12-02214(s) & MOC & $02 / 21 / 2000$ & 2.77 & $482 \times 598$ & Argyre \\
PSP_005596_1245(t) & HiRISE & $10 / 06 / 2007$ & 0.50 & $2,196 \times 864$ & Argyre \\
PSP_006163_1345(u) & HiRISE & $11 / 19 / 2007$ & 0.25 & $2,345 \times 2,606$ & Argyre \\
\hline
\end{tabular}

\section{Methods}

Aiming to detect dust devils tracks automatically, three methods were developed, each one being an improvement of the predecessor, until a high level of accuracy was reached. The methods we are proposing are mainly based on morphological operators and their construction in sequence intended to improve some aspects not solved with a high degree of robustness by the preceding method. The main feature characterizing the dust devil tracks is their elongated linear shape, that is, thin shapes of long extensions, normally darker than the surrounding terrain. Method 1 uses the classic top-hat transform for extracting the tracks and morphological granulometries to define their thickness dimensions. Method 2 intends to search directly in every direction for those structures, by integrating information provided by a radial closing transform with linear structuring elements. Finally, Method 3, based on path closings, intends to recover all the regions of those thin structures, that is, not only their more linear (straight) segments but also their curvilinear components.

\subsection{Method 1: Closing Top-Hat}

The first method starts with an initial filtering by median (3x3 mask) and a morphological area opening $\gamma_{\lambda}$, which is equivalent to the union of all openings $\gamma$ with the connected Structuring Elements (SE) $B$ whose size in number of pixels equals $\lambda$, that is $\gamma_{\lambda}=\underset{i}{\vee}\left\{\gamma_{B i} \mid B_{i}\right.$ with $\left.\operatorname{Area}\left(B_{i}\right)=\lambda\right\}$, where $\vee$ is the supremum operator. 
The size of the area was set empirically and varies with the image spatial resolution. Next, an initial binarization by Otsu's method [19] is applied and a morphological granulometric analysis with a SE disk is carried out. A granulometry can be defined as the family $\Gamma=\left(\phi_{\lambda}\right)_{\lambda \geq 0}$ of closings by scales $\lambda B=\{\lambda b \mid b \in B\}$ with $\lambda \geq 0$, and $B$ convex. The granulometric analysis is used to infer the radius of the $\mathrm{SE}$ to be applied in the filtering by the top-hat $\mu_{B}(f)=\phi_{B}(f)-f$, where $\phi_{B}(f)$ is the closing of $f$. The radius is chosen so that all dark components in the image do not fit the SE. Finally a binarization by Otsu is carried out to detect the features. The detection is based on the width of the tracks.

\subsection{Method 2: Radial Closing}

The second method starts with an initial filtering by a median operator ( $3 \times 3$ mask) and then applies a morphological radial closing [18]. This is the intersection of the closings performed with linear SEs aligned in every direction, that is $\psi(f)={ }_{i} \phi_{B i}(f)$. The angles considered vary from $i=0^{\circ}$ to $360^{\circ}$ in steps of $5^{\circ}$. The length of the SE was empirically chosen and varies with the spatial resolution of the images. Finally, a binarization by Otsu method is performed. The length of tracks is the feature considered in the detection.

\subsection{Method 3: Path Closing Top-Hat}

This third method starts with an initial filtering by morphological surface area opening and closing. The definition of surface area closing is obtained by duality from area opening. Next, a morphological path closing is applied. They can be defined by duality from de definition of path openings given by [20] as follows. Let $E$ be the image domain endowed with a binary adjacency relation $x \rightarrow y$. We call $x$ a predecessor of $y$ and $y$ a successor of $x$. Using the adjacency relation it is possible to define a dilation by writing $\delta(\{x\})=\{y \in E \mid x \rightarrow y\}$. The $L$-tuple $\boldsymbol{a}=\left(a_{1}, a_{2}, \ldots, a_{L}\right)$ is called a $\delta$-path of length $L$ if $a_{k} \rightarrow a_{k+1}$. The set of all $\delta$-path of length $L$ contained in a subset $X$ of $E$ is denoted by $\Pi_{L}(X)$. Then the path opening $\gamma_{L}$ is the union of all paths of length $L$ contained in $X$, and we can write $\gamma_{L}(X)=\bigcup\left\{\boldsymbol{a} \mid \boldsymbol{a} \in \Pi_{L}(X)\right\}$. This equation can be extended to gray level images by the principle of threshold decomposition [18]. The search for the paths is done in four directions $\left(0^{\circ}, 45^{\circ}, 90^{\circ}\right.$ and $135^{\circ}$ ) of the grid according to the rules defined by [20]. The lengths of the paths are defined by the diagonal length of the images times two (although the path closings being used are the constrained ones defined by [20], they still may zig-zag a little so the biggest possible path in the worse case would be the image diagonal times two). Next, the resulting images are binarized by Otsu method to detect the tracks. The detection is mainly driven by the length of the tracks.

\section{Results and Discussion}

The methods discussed in the previous section were applied to the images shown in Fig. 1. The results for two different spatial resolutions with the images 
PSP_002548_1255A and E13-00271(Figs. 1(d) and 1(m)) are shown in Figs. 2(a) to (c) and 3(a) to (c) for all three methods. The analysis of the results was made accordingly to the procedure proposed in [21] and is based on the following measurement Accuracy $=(T P+T N) /(m \times n)$, where $T P$ stands for true positives and $T N$ for true negatives. $T P$ and $T N$ are defined relative to a ground truth or reference image. For a processed image $P I$ and a ground truth image $G T, T P$ and $T N$ are calculated as $T P=\operatorname{Area}(G T \cap P I)$ and $T N=\operatorname{Area}(\sim G T \cap \sim P I)$, where $\cap$ and $\sim$ are the operators intersection and negation, respectively. For each of the 21 images processed, a ground truth image was made manually by an expert on a computer screen. As examples, the ground truth made for image PSP_002548_1255A is shown in Fig. 2(d) and the one made for image E13-00271is shown in Fig. 3(d). For image PSP_002548_1255A the accuracies were 0.7866 for method $1,0.8369$ for method 2 and 0.9414 for method 3 .

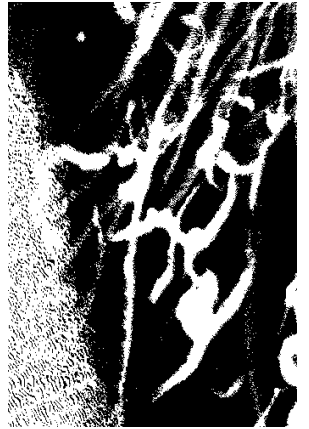

(a)

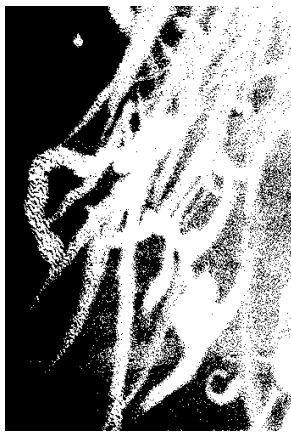

(b)

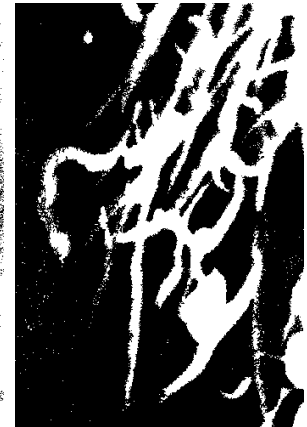

(c)

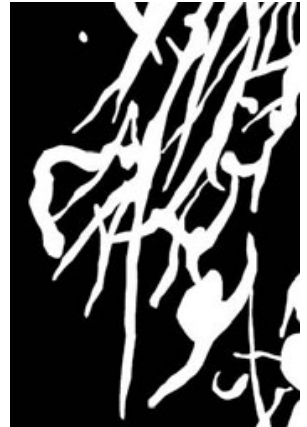

(d)

Fig. 2. Tracks detection in the image PSP_002548_1255A: (a) method 1, (b) method 2, (c) method 3 and (d) ground truth

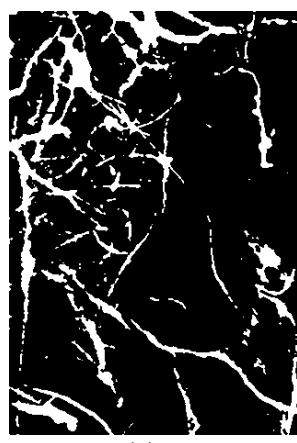

(a)

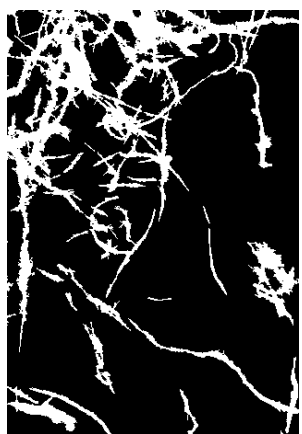

(b)

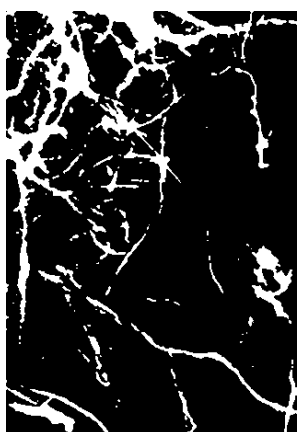

(c)

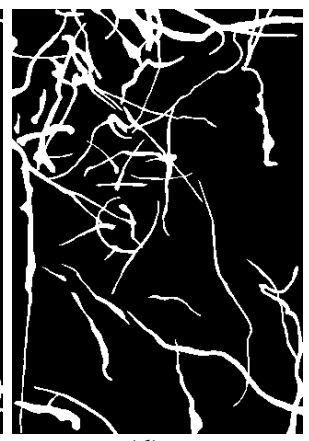

(d)

Fig. 3. Tracks detection in MOC image E13-00271: (a) method 1, (b) method 2, (c) method 3 and (d) ground truth 
And for image E13-00271 the accuracies were 0.9638, 0.9669 and 0.9612 for methods 1, 2 and 3, respectively. Table 2 summarizes statistics of the analysis for the whole set of images. Method 2 was the worse one with mean accuracy $0.8230 \pm$ 0.1196 . Besides, it is the one by which the smallest accuracy was reached $(0.5955$ for image M10-01206 (Fig. 1(h)). Methods 1 and 3 presented mean accuracies $0.8857 \pm$ 0.0660 and $0.8960 \pm 0.0770$, respectively. Considering their standard deviations there is no reason to believe that method 3 is better than method 1. Although, when it comes to the time of processing, method 3 is far faster (mean time of processing 96.27 $\mathrm{s} \pm 140.03 \mathrm{~s}$ per image against the $3,566.24 \mathrm{~s} \pm 7,625.67 \mathrm{~s}$ of method 1). Speed is an important factor to be considered, especially when working with larger files like those of the HiRISE images. Some of them may have hundreds of thousands of lines and in such cases method 3 would be the preferable for processing. This is why we assume it is the best method from the three presented here.

Table 2. Summary of the results obtained with the three methods

\begin{tabular}{ccccccc}
\hline & $\begin{array}{c}\text { Accuracy } \\
\text { Method 1 }\end{array}$ & $\begin{array}{c}\text { Accuracy } \\
\text { Method 2 }\end{array}$ & $\begin{array}{c}\text { Accuracy } \\
\text { Method 3 }\end{array}$ & $\begin{array}{c}\text { Time (s) } \\
\text { Method 1 }\end{array}$ & $\begin{array}{c}\text { Time (s) } \\
\text { Method 2 }\end{array}$ & $\begin{array}{c}\text { Time (s) } \\
\text { Method 3 }\end{array}$ \\
\hline Mean & 0.8857 & 0.8230 & 0.8960 & $3,566.24$ & 21.99 & 96.27 \\
Stdev & 0.0660 & 0.1196 & 0.0770 & $7,625.67$ & 29.80 & 140.03 \\
Min & 0.7567 & 0.5955 & 0.7099 & 6.93 & 0.68 & 4.1 \\
Max & 0.9781 & 0.9715 & 0.9732 & $30,461.22$ & 105.13 & 440.75 \\
\hline
\end{tabular}

\section{Conclusion}

The importance of studying Martian dust devils to get a better understanding of, for instance, low atmosphere and regolith characteristics, may be asserted by the huge amount of papers being published about the subject. But none of them proposes an automatic method for the detection of dust devils tracks. This has been done manually until now and is a time-consuming task. This paper presented three methods for detecting Martian dust devils tracks automatically. Each one is an improvement of its predecessor. All three are based on Mathematical Morphology. Method 3 was considered to be the best, not only for the high mean accuracy it produced but also for being the fastest, which is a crucial factor when processing HiRISE images that may have hundreds of thousands of lines. It succeeds in detecting tracks despite the variation in size and in spatial resolution of the images, and works for a great range of albedo variation as seen in Fig. 1, and can be a very useful tool for intensive mapping and largely increasing our understanding of these aeolion features. In particular, quantitative measures like width, length, number of tracks and their directions, among many others, can be carried out from the resulting binary images of our approach.

\section{References}

1. Balme, M.R., Whelley, P.L., Greeley, R.: Mars: Dust Devil Track Survey in Argyre Planitia and Hellas Basin. Journal of Geophysical Research 108 (2003)

2. Balme, M.R., Greeley, R.: Dust Devils on Earth and Mars. Reviews of Geophysics 44 (2006) 
3. Örmo, J., Komatsu, G.: Mars Orbiter Camera Observation of Linear and Curvilinear Features in the Hellas Basin: Indications for Multiple Processes of Formation. Journal of Geophysical Research 108 (2003)

4. Rennó, N.O.: A Thermodynamically General Theory for Convective Vortices. Tellus 60, 688-699 (2008)

5. Spiga, A., Forget, F., Lewis, S.R., Hinson, D.P.: Structure and Dynamics of the Convective Boundary Layer on Mars as Inferred from Large-Eddy Simulations and Remote Sensing Measurements. Quarterly Journal of the Royal Meteorological Society 136, 414-428 (2010)

6. Babak, T.G., Taylor, P.A.: Large Eddy Simulation of Typical Dust Devil-Like Vortices in Highly Convective Martian Boundary Layers at the Phoenix Lander Site. Planetary and Space Science 59, 43-50 (2011)

7. Lorenz, R.D., Jackson, B., Barnes, J.W.: Inexpensive Time-Lapse Digital Cameras for Studying Transient Meteorological Phenomena: Dust Devils and Playa Flooding. Journal of Atmospheric and Oceanic Technology 27, 246-256 (2009)

8. Hall, N.W., Lemmon, M.T.: Mass Ejected from Martian Dust Devils as a Function of Heigh and Dust Devil Morphology. In: XL Lunar and Planetary Sciences Conference, Texas, pp. 23-27 (2009)

9. Lorenz, R.D.: Experiments in Time-Lapse Camera Observations of Dust Devil Activity at Eldorado Playa, Nevada. In: XLII Lunar and Planetary Sciences Conference, Texas (2011)

10. Stanzel, C., et al.: Dust Devils Speeds, Directions of Motion and General Characteristics Observed by the Mars Express High Resolution Stereo Camera. Icarus 197, 39-51 (2008)

11. Lorenz, R.D.: Power Law of Dust Devil Diameters on Mars and Earth. Icarus 203, 684684 (2009)

12. Pathare, A.V., et al.: Assessing the Power Law Hypothesis for the Size-Frequency Distribution of Terrestrial and Martian Dust Devils. Icarus 209, 851-853 (2010)

13. Cantor, B.A., Kanak, K.M., Edgett, K.S.: Mars Orbiter Camera Observations of Martian Dust Devils and their Tracks (September 1997 to January 2006) and Evaluation of Theoretical Vortex Models. Journal of Geophysical Research 111, 1-49 (2006)

14. Whelley, P.L., Greeley, R.: The Distribution of Dust Devil Activity on Mars. Journal of Geophysical Research 113, 1-12 (2008)

15. Bridges, N.T., et al.: Aeolian Studies from HIRISE. In: XXXVIII Lunar and Planetary Science Conference, Texas (2007)

16. Bandeira, L., Saraiva, J., Pina, P.: Impact Crater Recognition on Mars Based on a Probability Volume Created by Template Matching. IEEE Transactions on Geoscience and Remote Sensing 45, 4008-4015 (2007)

17. Whelley, P.L., Greeley, R.: Latitudinal Dependency in Dust Devil Activity on Mars. Journal of Geophysical Research 111, 1-5 (2006)

18. Dougherty, E.R., Lotufo, R.A.: Hands-on Morphological Image Processing. SPIE, Washington (2003)

19. Otsu, N.: A Threshold Selection Method from Gray-Level Histograms. IEEE Transactions on Systems, Man and Cybernetics 9 (1979)

20. Hendriks, C.L.L.: Constrained and Dimensionality-Independent Path Openings. IEEE Transactions on Image Processing 19, 1587-1595 (2010)

21. Bandeira, L., Marques, J.S., Saraiva, J., Pina, P.: Automated Detection of Martian Dune Fields. IEEE Geoscience and Remote Sensing Letters 8, 626-630 (2011) 